$12-21-2021$

\title{
The "Ebb and Flow" of Documentation: Does the Transition Between Two Electronic Medical Records Systems Affect Emergency Department Efficiency?
}

\author{
Michelle Angeline MD \\ West Virginia University, michelle.angeline1@hsc.wvu.edu \\ Erica B. Shaver MD \\ West Virginia University, eshaver@hsc.wvu.edu \\ Christopher Kiefer MD \\ West Virginia University, ckiefer@hsc.wvu.edu \\ Kimberly D. Quedado PhD \\ kimberly.quedado@hsc.wvu.edu \\ Melinda Sharon \\ Marshall University
}

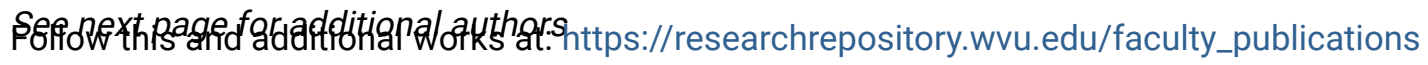

Part of the Health and Medical Administration Commons

\section{Digital Commons Citation}

Angeline, Michelle MD; Shaver, Erica B. MD; Kiefer, Christopher MD; Quedado, Kimberly D. PhD; Sharon, Melinda; Davis, Stephen PhD; Hurst, Kyle MD; Goode, Christopher S. MD; and Marshall, Thomas C. MD, "The "Ebb and Flow" of Documentation: Does the Transition Between Two Electronic Medical Records Systems Affect Emergency Department Efficiency?" (2021). Faculty \& Staff Scholarship. 3068. https://researchrepository.wvu.edu/faculty_publications/3068

This Article is brought to you for free and open access by The Research Repository @ WVU. It has been accepted for inclusion in Faculty \& Staff Scholarship by an authorized administrator of The Research Repository @ WVU. For more information, please contact beau.smith@mail.wvu.edu. 


\section{Authors}

Michelle Angeline MD, Erica B. Shaver MD, Christopher Kiefer MD, Kimberly D. Quedado PhD, Melinda Sharon, Stephen Davis PhD, Kyle Hurst MD, Christopher S. Goode MD, and Thomas C. Marshall MD 
3 ABSTRACT

4 Background: Electronic Medical Record (EMR) systems are electronic databases for compiling

5 patient records. As healthcare networks expand, it is critical for providers to have access to

6 patient data more broadly. As a result individual healthcare facilities must adjust to enterprise

7 wide EMRs.

8 Objective: This study examined the operational effects of transitioning from an Emergency

9 Department (ED) specific EMR to an enterprise wide EMR by evaluating throughput metrics in a 10 community ED.

11 Methods: During a 6-month transition period (July-December 2017) in a community-based,

12 academic ED located in North Central West Virginia, length of stay (LOS) and the following

13 operational metrics were analyzed: door-to-provider times, door to disposition time, average

14 LOS, left without treatment (LWOT) rates, and total ED volumes. These metrics were compared

15 with the prior year's same 6-month period to account for seasonal variability in patient pathology

16 or ED volumes.

17 Results: Overall, there was a statistically significant increase in the LOS measures, including

18 door-to-provider time $(\mathrm{p}=0.0003)$, door to disposition time $(\mathrm{p}<0.0001)$, average LOS $(\mathrm{p}<0.0001)$, 19 and LWOT $(\mathrm{p}=0.0008)$ rates in the post-implementation period. Of note, the overall ED volumes

20 post-implementation were significantly lower than pre-implementation $(\mathrm{p}<0.0001)$, further

21 supporting that EMR transition negatively impacted ED efficiency despite lower patient

22 volumes. 
24 Conclusion: An ED-specific EMR to enterprise wide EMR transition in a community ED had a

25 negative effect on the overall efficiency of the emergency department.

27 Key Words: Electronic Medical Record (EMR), Emergency Medicine, Healthcare

28 Administration

30 INTRODUCTION

\section{Background}

32 An effective emergency department (ED) triages, assesses, resuscitates, diagnoses, and

33 dispositions the most vulnerable and undifferentiated patients the most efficient manner possible.

34 Time management is a critical element in this process. As a result, the Emergency Medicine

35 (EM) physician is faced with the competing interests of providing effective and compassionate

36 patient care, and dealing with inevitable distractions and interruptions, all while being challenged

37 to master timely and complete documentation in the electronic medical record (EMR).

38 Importance

39 Historical data has shown that EMR implementation generally has a positive financial

40 return on investment (ROI) and leads to improved quality of patient care and safety.(1,2)

41 However, despite the many known benefits of the EMR, a recent study has called to question the

42 impact of the EMR on ED workflow, efficiency, and accuracy of documentation.(2)

43 Additionally, this research has started to objectively evaluate length of stay measures and

44 operational characteristics, both prior to and after implementation of an EMR to assess for

45 impact. Ward et. al published one of the largest studies to assess the effect of EMR

46 implementation on the operational metrics of a diverse group of community EDs.(2) While the 
47 study showed no meaningful difference in eight measures of operational performance, there were

48 some variations and negative impact on efficiency and operational metrics of some of the

49 individual EDs, sparking future work to assess which specific variables account for decreased

50 efficiency related to EMR implementation.

51 Recently, the transition from paper documentation to the use of an EMR has posed new

52 challenges for physicians when it comes to performing their duties in an efficient manner. This

53 has specifically had a profound effect on EM physicians, as they adapt to new routines while

54 maintaining optimal patient care as emergency department volumes increase.(3-6) The

55 introduction of the EMR as a means for more robust health information documentation processes

56 has posed frequent discussions regarding the benefits and risks of this transition. Prior literature,

57 as it applies specifically to ED operations and efficiency, has debated whether the advent of the

58 EMR helps or hinders EDs that have embraced the practice of electronic documentation. $(2,4-8)$

59 While the referenced studies do not focus on a transition between EMR systems, most studies

60 have demonstrated a largely negative effect on ED performance in the time period following

61 initial EMR introduction from paper documentation. These studies specifically reference

62 negative trends in the length of stay (LOS) and operational metrics, with one study

63 demonstrating an overall LOS increase of nearly 40 minutes within the first 4 weeks of EMR

64 implementation. $(2,4-6,8)$

65 As the healthcare system becomes increasingly complex, and accurate communication

66 and medical documentation across all specialties and services within a hospital system becomes

67 inevitably more vital, enterprise wide EMR's are gaining traction as a vehicle for improving the

68 overall quality and safety of patient care. 


\section{Goals of this Investigation}

71 While most previous studies have analyzed the transition from paper documentation to

72 initial ED implementation of an EMR, this study is the first, to our knowledge, to evaluate the

73 effect of the transition from an ED-specific EMR to an enterprise-wide EMR, and the subsequent

74 effects on length of stay and operational metrics in a community ED. We hypothesized that the

75 transition between EMRs would likely increase length of stay and other operational metrics

76 including door-to-provider times and left without treatment rates (LWOTs). The purpose of this

77 study was to measure the change in efficiency pre- and post - EMR deployment.

\section{METHODS}

\section{Study Design and Setting}

81 We conducted a longitudinal analysis of data from a single, community-based, university

82 affiliated ED located in North-Central West Virginia, with an annual volume of 58,000 patients

83 per year. The ED contains 36 acute treatment rooms and 292 licensed inpatient hospital beds.

84 The ED is staffed by board-certified and/or board-eligible Emergency Physicians. EM and

85 family medicine residents, nurse practitioners, and physician assistants serve as additional

86 providers at variably scheduled times. The study site utilized an ED-specific EMR for seven

87 years before transitioning to an enterprise-wide EMR in July 2017. During the two months prior

88 to full implementation of the new EMR, healthcare providers underwent 8 hours of standardized

89 EMR training that was uniformly implemented across the health system. For the first two weeks

90 of deployment in July 2017, specially certified users were on site and available to local

91 providers. After this immediate post implementation period EMR support was available via

92 phone. 
In order to quantify the impact of EMR transition, this study analyzed length of stay and

94 operational characteristics, with the primary outcome being average ED LOS. Secondary

95 outcomes for analysis included door-to-provider times and left without treatment (LWOT) rates.

96

\section{Measurements \& Analysis}

Data from two discrete 6-month time periods was analyzed: the first representing a 6-

99 month period (July 1-December 31, 2016) preceding implementation of the enterprise EMR by

100 exactly one year, and the second representing the same time period post-EMR transition (July 1-

101 December 31, 2017). The same 6-month time frame of each year was chosen to control for

102 seasonal variations in ED volumes and patient pathology.

103 The primary outcome of the study was total LOS. The extent to which the primary

104 outcome departed from normality was assessed via the Shapiro-Wilk test. A One-way Analysis

105 of Variance (ANOVA), or the nonparametric Kruskal-Wallis test in cases of non-normal

106 outcomes, was used to test whether or not average LOS differed after the EMR change.

107 Secondary outcomes of our study included door-to-provider times and left without treatment

108 (LWOT) rates during the two study periods. An alpha of 0.05 was selected as the threshold for

109 statistical significance. All analyses were conducted using JMP® 13.2.1 (SAS Institute, Inc).

\section{RESULTS}

111 From July 1 to December 31, 2016, there were a total of 29,480 patients (1,095 average

112 per week) who presented to the ED. During this initial time period under the ED-specific EMR,

113 the average total LOS was 176 minutes (Figure 1). Average door-to-provider time was 41.2

114 minutes, with a LWOT rate of $1.97 \%$. Following implementation of the enterprise-wide EMR,

115 from July 1 to December 31,2017 , there were a total of 27,711 patients $(1,026$ average per 
116 week) who presented to the ED, a statistically significant decrease in patient volume compared to 117 the prior similar time period $(\mathrm{p}<0.0001)$. Post-EMR transition, data showed a significant increase 118 in the average LOS to 207 minutes, an overall increase of 31 minutes $(\mathrm{p}<0.0001$; Figure 1).

119 Door-to-provider time and LWOT rates both significantly increased to 53.2 minutes and $3.81 \%$, 120 respectively $(\mathrm{p}<0.0001$; Figure 2$)$.

121 Figure 1. Average length of stay, pre- and post-implementation.

122

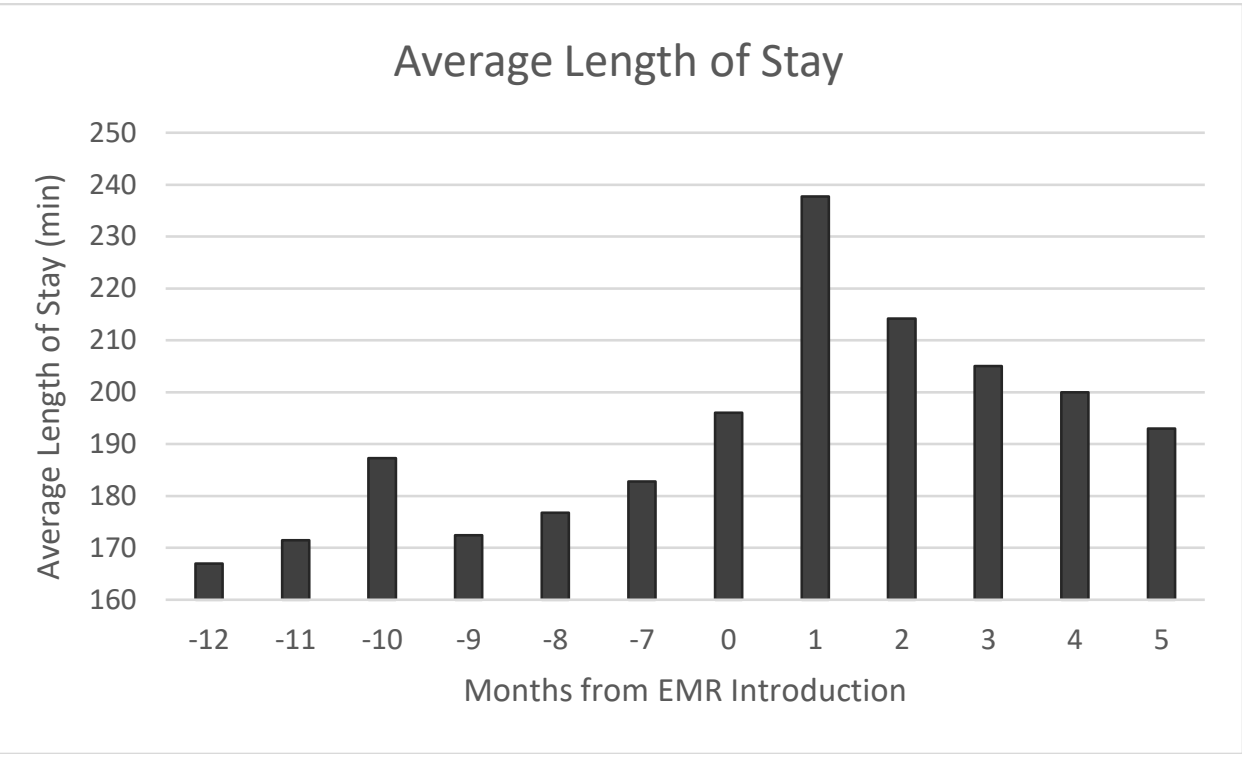

Note: Scale provides month (July 2016 to December 2016) to month (July 2017 to December 2017) comparison of pre and post EMR implementation to correct for seasonal variation. Data

Figure 2. Average door-to-provider times, pre- and post-implementation. 


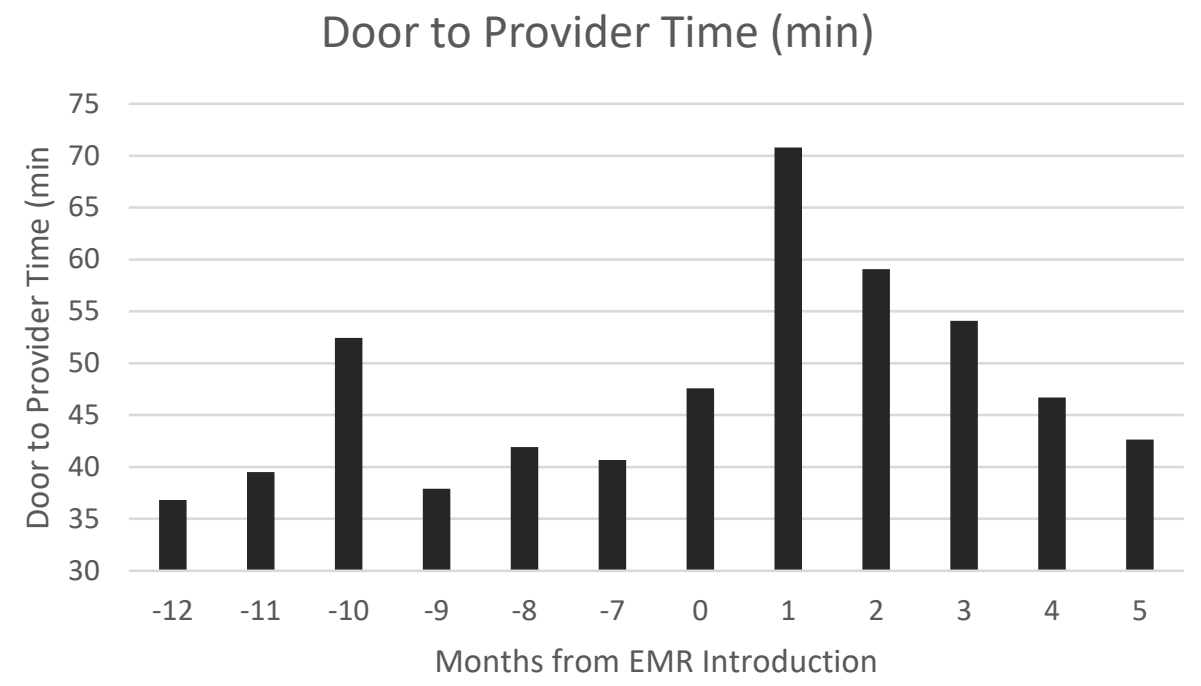

Note: Scale provides month (July 2016 to December 2016) to month (July 2017 to December 2017) comparison of pre and post EMR implementation to correct for seasonal variation. Data points are expressed as weekly averages during the entirety of the study.

Figure 3. Average percentage left without treatment rates, pre- and post-implementation.

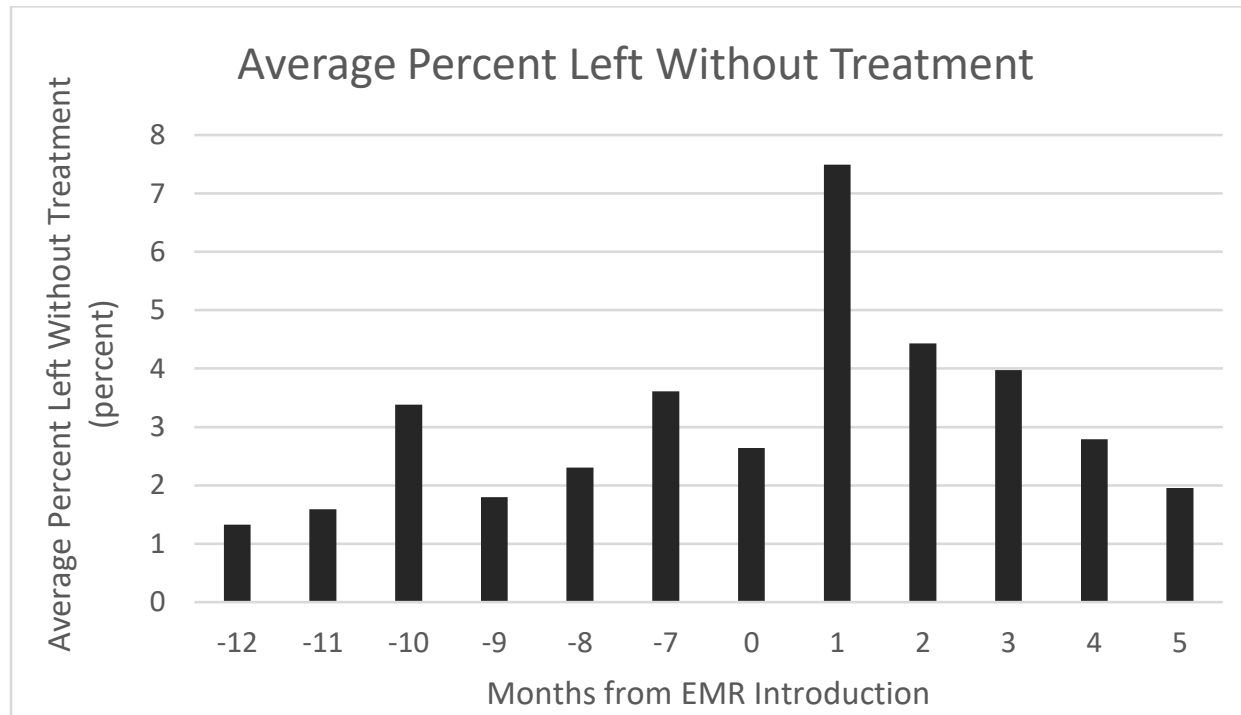

Note: Scale provides month (July 2016 to December 2016) to month (July 2017 to December 2017) comparison of pre and post EMR implementation to correct for seasonal variation. Data 
To our knowledge, this is the first study to analyze specific length of stay and operational

148 metrics following a transition between EMR systems. Most of the prior literature has analyzed

149 the transition from paper medical records to an EMR. Our results from an ED specific EMR

150 system to enterprise wide EMR transition were consistent with prior paper to EMR transition

151 studies that have demonstrated a negative impact on ED efficiency metrics immediately after

152 implementation of a new EMR.(2-6). We found significant increases in average length of stay,

153 door to provider times, and left without treatment rates. We did attempt to control for

154 confounding factors such as annual seasonal variability by studying the same time periods per

155 and post EMR implementation. Our results indicated an increase of 31 minutes to total length of

156 stay post-implementation of the enterprise-wide EMR. The fact that the total patient volumes

157 were incidentally lower immediately post implementation makes these increases in length of stay

158 even more striking, as patient length of stay times likely would have been further increased had

159 patient volumes been similar to the same time period pre-enterprise-wide EMR implementation.

160 Our results differed from one of the largest prior studies analyzing the paper to EMR

161 transitions and the effect upon ED efficiency. Ward et al. found no difference in length of stay

162 measures and operational characteristics in the overall analysis of 23 community EDs after these

163 EMR implementations.(2) We believe our results may have differed for multiple reasons. First,

164 our study evaluates a single clinical site with a fully encompassing EMR, including registration,

165 provider documentation, order entry, diagnostic processing and resulting, as well as integration

166 of bed requests and admission orders while the prior work evaluated a variety of sites, with

167 variable EMR penetration across the clinical setting.(8)

168 With more independent community hospitals being incorporated into larger health

169 systems, we anticipate these enterprise-wide EMR transitions to become extremely common. 
170 Over the past decade, health systems having increasingly adopted EMR systems, citing potential

171 cost savings, with prior estimates revealing a potential nationwide savings of \$162 billion

172 through increased safety and system efficiency.(9) Given this, system-wide EMR consolidation

173 to a single platform is increasingly favored by the leadership of these health systems, as they

174 believe it establishes a secure way to share HIPAA-sensitive data, thereby improving

175 communication, decreasing duplicate testing within the same system, and increasing patient

176 safety.(10) Unfortunately, current EMR models vary widely in their interface, content, and

177 operability, which can make it difficult for clinicians to seamlessly transition from one EMR

178 system to another.(11) This likely helps to explain the negative operational effects observed in

179 our study following the transition from one EMR system to another.(11)

180 Limitations of this study include that data was obtained from a single site, community

181 ED. In addition, the post-implementation time period used in the analysis was the six months

182 immediately following the change in EMR, which differed somewhat from the data presented by

183 Ward et al which utilized a "steady state" period following EMR implementation as the post

184 intervention comparison period. We were unable to evaluate a "steady state" period, as the

185 negative effects on patient throughput necessitated a significant workflow change that involved

186 implementation of a new "split-flow" process. Thus, we are lacking the data to evaluate whether

187 or not our location had a return to baseline in levels of efficiency after a time period directly after

188 implementation had passed and steady state had returned.

189 Additionally, during the study period, all aspects of the hospital in the study, including

190 inpatient wards, pharmacy, operating rooms, laboratory services, and radiology were

191 transitioning from various forms of documentation to the enterprise-wide EMR. Although these

192 changes can also significantly affect ED operational efficiency, we did not quantify the impact of 
193 these individual units or the differing levels of EMR experience of the providers. As a result of

194 these variables, it is difficult to distinguish exactly where delays occurred.

195 This study illustrates the need for EDs to adequately prepare for an EMR transition. By

196 working with institutional Information Technology in order to ensure adequate training for

197 providers, potentially increasing provider staffing during the transition, and making on-site

198 technical support available during the transition for issues that arise during clinical care, this can

199 hopefully mitigate an anticipated decline in operational efficiency and performance metrics.

200

201

202

203

\section{References}

204

1. Wang SJ; Middleton B; Prosser LA; Bardon CG; Spurr CD; Carchidi PJ, et al. A cost-benefit

205

206

207

208

209

210

211

212

213

214

215

216

217

218

219

220

221

222

223

224 analysis of electronic medical records in primary care. Am J Med. 2003;114(5):397-403.

2. Ward MJ; Landman AB; Case K; Berthelot J; Pilgrim RL; Pines JM. The effect of electronic health record implementation on community emergency department operational measures of performance. Ann Emerg Med. 2014;63(6):723-30.

3. Feblowitz J; Takhar SS; Ward MJ; Ribeira R; Landman AB. A Custom-Developed Emergency Department Provider Electronic Documentation System Reduces Operational Efficiency. Ann Emerg Med. 2017;70(5):674-82.e1.

4. Perry JJ; Sutherland J; Symington C; Dorland K; Mansour M; Stiell IG. Assessment of the impact on time to complete medical record using an electronic medical record versus a paper record on emergency department patients: a study. Emerg Med J. 2014;31(12):980-5.

5. Mohan MK; Bishop RO; Mallows JL. Effect of an electronic medical record information system on emergency department performance. Med J Aust. 2013;198(4):201-4.

6. Park SY; Lee SY; Chen Y. The effects of EMR deployment on doctors' work practices: a qualitative study in the emergency department of a teaching hospital. Int J Med Inform. 2012;81(3):204-17.

7. Farley HL; Baumlin KM; Hamedani AG; Cheung DS; Edwards MR; Fuller DC, et al. Quality and safety implications of emergency department information systems. Ann Emerg Med. 2013;62(4):399-407.

225

8. Ward MJ; Froehle CM; Hart KW; Collins SP; Lindsell CJ. Transient and sustained changes in operational performance, patient evaluation, and medication administration during 
electronic health record implementation in the emergency department. Ann Emerg Med. 2014;63(3):320-8.

2289 9. Hillestad R. Health care IT adoption could save USD162 billion. World Hosp Health Serv. 229 2006;42(2):36, 8-40.

230 10. Arndt; Z. R. M\&A drives health systems' EHR consolidation. Modern Healthcare [serial on the Internet]. 2018 January 28, 2019: Available from: https://www.modernhealthcare.com/article/20180403/NEWS/180409973/m-a-driveshealth-systems-ehr-consolidation.

11. Kellermann AL; Jones SS. What it will take to achieve the as-yet-unfulfilled promises of health information technology. Health Aff (Millwood). 2013;32(1):63-8. 